\title{
Controle físico-químico do camarão salgado-seco comercializado no Estado do Rio de Janeiro
}

\author{
Chemical-physical control in dried-salted shrimp comercialized \\ in Rio de Janeiro State
}

\author{
Fabiana Bom Kraemer, ${ }^{\star}$ Jussara Schwind Pedroso Stussi,, ${ }^{\star *}$ Nelson Nebel Santos,, ${ }^{\star \star *}$ Diana Bridon da Graça Sgarbi, ${ }^{\star \star}$ \\ Sérgio Carmona de São Clemente***
}

\begin{abstract}
Resumo
O presente trabalho determinou os teores de umidade, resíduo mineral fixo, cloreto de sódio ( $\mathrm{NaCl}$ ) e o número de Ácido Tiobarbitúrico (TBA) em camarão salgado-seco obtido de diversas lojas comerciais e feiras livres do Estado do Rio de Janeiro, Brasil. As determinações foram realizadas de modo a avaliar a qualidade do produto frente a essas características, encontrando-se os seguintes valores médios: umidade $48,7 \%$, resíduo mineral fixo $25,9 \%, \mathrm{NaCl} 20,1 \%$ e TBA 0,54. É importante destacar que todas as amostras ultrapassaram o limite para umidade estabelecido pela legislação brasileira para pescado (35\%), donde conclui-se a necessidade de fiscalização, a fim de evitar alterações indesejáveis do produto com prejuízo à saúde da população.
\end{abstract}

Palavras-chave: camarão salgado-seco; qualidade; controle físico-químico.

\begin{abstract}
This communication reports moisture content, ash, sodium cloride and thiobarbituric acid number (TBA) in dried-salted shrimp collected at markets and retail shops in Rio de Janeiro State, Brazil. The mensurations were made to evaluate the quality of these products considering those characteristics. Mean values were: moisture 48,7\%, ash $25,9 \%, \mathrm{NaCl} 20,1 \%$ and TBA 0,54 . It's important to observe that all samples exceed the limit allowed for Brazilian legislation for moisture content (35\%) . We can concluded the necessity to monitorize this product in order to prevent the sale of na improper product.
\end{abstract}

Keywords: dried-salted shrimp; quality; chemical-physical control.

\section{Introdução}

O camarão constitui uma importante fonte protéica na dieta de porção significante da população, principalmente, em países tropicais. A composição de ácidos graxos dos lipídios totais [hepatopâncreas e músculo] do camarão, Penaeus japonicus, é semelhante à de outros animais marinhos. Os principais ácidos graxos encontrados são o palmítico, o esteárico, o olêico, o eicosapentaenóico (EPA) e o docosahexaenóico (DHA). No músculo do camarão encontram-se, principalmente, fosfolipídios e colesterol livre (Muriana et al., 1993; Muriana, Ruiz-Gutierrez, Bolufer, 1993).

Dentre os produtos do pescado salgado, encontramos o camarão salgado-seco, cujo consumo é popular no Brasil, principalmente no nordeste do país, onde o produto é oriundo de pesca artesanal (Rogers e Sanders, 1992).
Em alimentos, a reação mais importante ocorre com os lipídios insaturados, que consiste na oxidação pelo oxigênio do ar, também denominada autoxidação (Bobbio e Bobbio, 1989), sendo incriminados à ação da luz, do calor, do oxigênio e das enzimas autolíticas ou de microrganismos como causas das alterações de natureza química nos lipídios (Farchmin, 1967).

A ingestão de lipídios oxidados, ácidos graxos peroxidados, polímeros e esteróis oxidados, substâncias potencialmente tóxicas, podem contribuir para a ocorrência de processos como arteriosclerose e, possivelmente, o câncer (Araújo, 1995 e Ferrari, 1998).

Os fosfolipídios constituídos de ácidos graxos poliinsaturados (AGPI) com mais de quatro ou cinco ligações duplas como os EPA e DHA, que possuem seis e sete ligações duplas, respectivamente, são mais susceptíveis à oxidação que os lipídios

\footnotetext{
* Departamento de Nutrição Aplicada - Instituto de Nutrição - UERJ. Rua São Francisco Xavier, 524/12 andar, sala 12030/D - CEP 20550-013 Maracanã - Rio de Janeiro, RJ.

** Departamento de Microbiologia e Parasitologia - Instituto Biomédico - UFF - Niterói, RJ.

*** Departamento de Tecnologia de Alimentos - Faculdade de Veterinária - UFF - Niterói, RJ.
} 
constituídos de ácidos graxos saturados, monoinsaturados ou diinsaturados. Os peróxidos formados nesta oxidação são decompostos até a formação de produtos finais estáveis (aldeídos, cetonas, ésteres, álcoois, ácidos, hidrocarbonetos etc), geralmente tóxicos (Ferrari, 1999).

De todos os produtos de origem animal, o pescado é o mais sensivel à autólise, à oxidação, à hidrólise de gorduras e à alteração por microrganismos (Frazier e Westhoff, 1993). Como conseqüência, aplicam-se, para sua conservação, métodos rápidos de tratamento, entre os quais podemos incluir a salga (Möhler, 1982).

A susceptibilidade à autoxidação em produtos salgado-seco e salgado-defumado dependem do conteúdo de umidade, porosidade do tecido, conteúdo de sal e permeabilidade ao oxigênio e vapores de água dos materiais utilizados para a embalagem (Akande, Knowles e Taylor, 1991).

De acordo com o parágrafo único do artigo 465 do Regulamento da Inspeção Industrial e Sanitária de Produtos de Origem Animal (RIISPOA) (Brasil, 1997), o pescado salgado-seco não deve conter mais de $35 \%$ de umidade, nem mais de $25 \%$ de resíduo mineral fixo total. Comercializado, também, em condições nem sempre ideais, o camarão salgado-seco pode sofrer alterações microbianas e físico-químicas, com reflexo direto sobre o consumidor. Em razão disso, para que os alimentos in natura e demais derivados sejam conservados de maneira apropriada para o consumo é indispensável que sejam assegurados proteção e controle, quanto ao acesso dos fatores causais de tais alterações indesejadas. É, portanto, importante a mensuração de determinadas características físico-químicas que poderiam ser resultantes de conservação inadequada, do processamento ou das condições de comercialização desse produto, no Estado do Rio de Janeiro.

\section{Materiais e métodos}

Foram analisadas 20 amostras de camarão salgado-seco, de aproximadamente $500 \mathrm{~g}$ cada uma, adquiridas no comércio varejista do Estado do Rio de Janeiro, Brasil, sendo transportadas ao Laboratório de Controle Físico-Químico de Produtos de Origem Animal da Faculdade de Veterinária da Universidade Federal Fluminense, conservados à temperatura ambiente $26 \pm 2{ }^{\circ} \mathrm{C}$, em suas embalagens comerciais originais. Unidades analíticas de $100 \mathrm{~g}$ da amostra foram trituradas em liqüidificador (OSTERIZER) até homogeneização completa do produto, sendo posteriormente analisadas em duplicata, como é descrito a seguir.

A determinação do percentual de umidade foi realizada através de adaptação do método gravimétrico pelo processo de aquecimento em estufa a $105^{\circ} \mathrm{C}$, descrito pelo Laboratório Nacional de Referência Animal (LANARA) (Brasil, 1981).

O teor de cloreto de sódio ( $\mathrm{NaCl}$ ) foi determinado volumetricamente pelo método de Mohr (Brasil, 1981).

A determinação do número do TBA foi realizada de acordo com adaptação da técnica descrita por Tarladgis et al. (1960). A leitura dos resultados foi realizada com o auxílio de um espectrofotômetro (MICRONAL - B280) num comprimento de onda de 538 nanômetros e os resultados obtidos foram padronizados de acordo com a técnica utilizada; ou seja, multiplicado pelo fator 7,8 para converter em $\mathrm{mg}$ de malonaldeído por $1000 \mathrm{~g}$ de amostra (número de TBA).

\section{Resultados e discussão}

$\mathrm{Na}$ determinação da umidade das 20 amostras de camarão salgado-seco, encontraram-se valores entre $40,4 \%$ e $55,5 \%$ com média (e desvio-padrão) de $48,7 \% \pm 3,9$. Todas as amostras apresentaram teor de umidade acima do permitido pelo RIISPOA (Brasil, 1997), sugerindo que as amostras não foram devidamente secas ou sua estocagem não foi feita de modo apropriado.

O processo de salga e dessecação é considerado o mais comum e barato método de conservação de pescado, sendo um produto elaborado de forma artesanal pelo pescador/produtor como forma de fazer chegar ao consumidor um alimento com qualidades nutricionais e higiênico-sanitárias adequadas para o consumo (Rogers e Sanders, 1992). Observou-se, no momento da aquisição das amostras, desrespeito às normas higiênico-sanitárias, com condições deficientes na comercialização. Em feiras livres, o produto é comercializado a granel, exposto em tabuleiros de madeira e separado em porções, utilizando-se latas de óleo vazias, sendo vendido o "litro". Na mesma bancada, são expostos produtos outros como carnes frescas e produtos de origem vegetal. Nos mercados e mercearias, o produto é comercializado a granel ou pré-embalado, em embalagens do próprio estabelecimento, sendo mantidos, em alguns casos, próximos a produtos de origem vegetal e podendo-se, ainda, verificar que determinados recipientes apresentavam umidade no fundo, indicativo de terem sido mantidos sob refrigeração até o momento da venda. Essa falta de cuidado na armazenagem e/ ou comercialização poderia, em parte, explicar um possível aumento do teor de umidade do produto.

As concentrações de resíduo mineral fixo nas amostras analisadas variaram entre $18,1 \%$ e $33,0 \%$ e a de $\mathrm{NaCl}$, entre 11,7 $\%$ e $28,7 \%$. As concentrações (\%) médias (e desvio-padrão) foram de $25,9 \pm 4,0$ para resíduo mineral fixo e $20,1 \pm 4,3$ para $\mathrm{NaCl}$. A regressão linear para o cloreto em função de cinza apresentou um $\mathrm{R}^{2}=0,97$.

Verificou-se. ainda, que 55\% das amostras estão acima do limite estabelecido pelo RIISPOA (Brasil, 1997) para resíduo mineral fixo. $\mathrm{E}$, ainda, que sua correlação com o $\mathrm{NaCl}$ foi alta, o que significa dizer que existe uma grande dependência entre elas.

As concentrações de malonaldeído nas amostras analisadas nesse estudo variaram de 0,00 a 1,24 , com média e respectivo desvio-padrão de $0,54 \pm 0,3$.

Os valores de TBA encontrados estão dentro do limite aceitável quando comparado com o sugerido por Shinhuber e $\mathrm{Yu}$ (1958), até 3 números de TBA. Entretanto, a avaliação deste dado torna-se difícil por não se dispor de informação acerca do tempo de armazenamento do camarão analisado, dado que influencia diretamente o valor de TBA segundo Akande, Knowles e Taylor (1991), que verificaram uma redução do conteúdo de malonaldeído em pescado salgado e armazenado a $30^{\circ} \mathrm{C}$ por 20 semanas, e Smith, Hole e Hanson (1990), que observaram diminuição após 10 semanas de armazenamento. Segundo os mesmos autores, isso pode ocorrer devido ao fato do malonaldeído reagir com outras substâncias, diminuindo seu conteúdo com o tempo de armazenamento.

Os lipídios do pescado são muito susceptíveis à oxidação pelo ar atmosférico, possuindo o pescado seco pouca prote- 
ção e tendo, possivelmente, o sal como catalisador dessa reação (Maruf et al., 1990). Esses mesmos autores verificaram que o aumento no conteúdo de sal da salmoura de pescado, de $5 \%$ para $15 \%$, resultou em aumento dos valores de TBA, fato este não observado no presente trabalho, onde os maiores valores de TBA $(1,17$ e 1,24$)$ não são os que apresentam maiores conteúdos de $\mathrm{NaCl}$.

\section{Conclusão}

Todas as amostras avaliadas apresentaram teor de umidade acima do determinado pelo RIISPOA (Brasil, 1997) para esse tipo de produto; e $55 \%$ das amostras estão acima do limite estabelecido, pelo mesmo regulamento, para o resíduo mineral. Esses valores demonstram que o processamento

\section{Referências}

AKANDE, G. R., KNOWLES, M. J., TAYLOR, K. D. A. Rancidity and changes in fatty acid composition of salted dried and salted smoked mackerel cakes. Trop. Sci., v. 31, p. 55-63, 1991.

ARAÚJO, J. M. A. Química de alimentos: teoria e prática. Minas Gerais: Imprensa universitária, Universidade Federal de Viçosa, 1995.

BOBBIO, F. O., BOBBIO, P. A. Introdução à química de alimentos. 2 . ed. São Paulo: Varela, 1989.

BRASIL. Ministério da Agricultura e do Abastecimento. Secretaria Nacional de Defesa Agropecuária. Laboratório Nacional de Referência Animal [LANARA] Métodos Analíticos Oficiais para Controle de Produtos de Origem Animal e seus ingredientes. II Métodos Físicos e Químicos. Brasília, DF, 1981.

Ministério da Agricultura e do Abastecimento. Secretaria de Defesa Agropecuária. Departamento de Inspeção de Produtos de Origem Animal. Regulamento da inspeção industrial e sanitária de produtos de origem animal. Aprovado pelo Decreto $n^{\circ} 30.691,29 / 3 / 52$, alterado pelos Decretos $n^{\circ} 1255$ de 25/6/62, 1236 de 2/9/94, 1812 de 8/2/96 e 2244 de 4/6/97. Brasília, DF, 1997.

FARCHMIN, G. Inspección veterinaria de alimentos. Zaragoza: Acribia, 1967.

FERRARI, C. K. B. Oxidação lipídica em alimentos e sistemas biológicos: mecanismos gerais e implicações nutricionais e patológicas. $R e-$ vista de Nutrição, v. 11, n. 1, p. 3-14, 1998.

Oxidação de lipídios e antioxidantes: importância nas ciências animal e dos alimentos. Higiene Alimentar, v. 13, n. 60, p. 16-20, 1999. tecnológico e/ou as condições de comercialização utilizados na produção do camarão salgado-seco não são adequados.

Noventa e cinco por cento das amostras apresentou valor de TBA diferente de zero, o que poderia ser indicativo de ranço oxidativo.

O camarão salgado-seco deve ser avaliado quanto à evolução dos valores de TBA, a fim de se evitar o consumo de produtos de qualidade duvidosa frente ao problema do ranço oxidativo.

Os dados obtidos mostraram ser necessária a fiscalização do camarão salgado-seco com especial atenção às normas higiênico-sanitárias, ao índice de umidade e cinzas, pois grande parte das amostras estavam fora do padrão nacional estabelecido.

FRAZIER, W. C., WESTHOFF, D. C. Microbiología de los alimentos. Tradução de Manuel Ramis Vergés. 4. ed. Zaragoza: Acribia, [1993]. 681 p. Tradução de: Food Microbiology.

MARUF, F. W., LEDWARD, D. A., NEALE, R. J., POULTER, R. G. Chemical and nutricional quality of Indonesian dried-salted mackerel (Rastrelliger kanagurta). International Journal of Food Science and Technology, $v$. 25, n. 1, p. 66-77, 1990.

MÖHLER, K. El curado. Tradução de Jaime Esaín Escobar. Zaragoza: Acribia, [1982].

MURIANA F. J. et al. A study of the lipids and carotenoprotein in the prawn, Penaeus japonicus. J Biochem, v. 114, n. 2, p. 223-229, 1993.

RUIZ-GUTIERREZ, V., BOLUFER, J. Phospholipid fatty acid compositions of hepatopancreas and muscle from the prawn, Penaeus japonicus. J Biochem, v. 114, n. 3, p. 404-407, 1993.

ROGERS, J. F., SANDERS, A. O pescado salgado-seco no Maranhão: diagnóstico e perspectivas. São Luís: EDUFMA, 1992.

SINHUBER, R. O., YU, J. C. 2-Thiobarbituric acid method for the measurement of rancidity in fishery products II. The qualitative determination of malonaldehyde. Food Technology, v. 12, p. 9-12, 1958.

SMITH, G., HOLE, M. HANSON, S.W. Assessment of Lipid Oxidation in Indonesian Salted-dried Marine Catfish (Arius thalassinus). Journal of the Science of Food and Agriculture, v. 51, n. 2, p.193205, 1990.

TARLADGIS, B.G. et al. Distillation method for the quantitative determination of malonaldehyde in rancid foods. Journal of American Oil Chemistry Society, v. 37, p. 44-48, 1960. 